\title{
Spin-orbit coupling in biradicals: Structural aspects
}

\author{
Josef Michl and Zdenхk Havlas \\ Department of Chemistry and Biochemistry, University of Colorado, Boulder, CO \\ 80309-0215, U. S. A., and Institute of Organic Chemistry and Biochemistry, Academy \\ of Sciences of the Czech Republic, 16610 Prague 6, Czech Republic
}

Abstract: Structural dependence of spin-orbit coupling in biradicals is discussed in non-mathematical terms. The importance of through-bond coupling and the origin of substituent effects, in particular, heavy atom effects, are discussed and illustrated.

\section{INTRODUCTION}

In many triplet photochemical reactions in condensed media, $T_{1}$ to $S_{0}$ intersystem crossing (ISC) is involved in the rate and/or product determining step (e.g., refs. 1,2). A good intuitive understanding of its rate as a function of molecular structure and conformation is clearly desirable. Such understanding requires two types of information: (i) which geometries is the triplet molecule likely to visit as it roams the $T_{1}$ surface, and (ii) how likely is the ISC at each such geometry. The former is dictated by the nature of the initial excitation, the temperature, and the shape of the $T_{1}$ surface. The ISC step often occurs at a biradicaloid geometry. The latter type of information is of interest to us presently. E.g., the introduction of a heavy atom usually does not greatly affect potential energy surface shapes, but it may greatly enhance the ISC rate, or it may have nearly no effect (e.g., ref. 3), or it may even reduce the ISC rate (ref. 4).

When the two radical centers of a biradical are well separated, hyperfine interactions provide the main ISC mechanism (e.g., ref. 5). When they are close together, spin-orbit coupling (SOC, ref. 6) is believed to play a dominant role. The usual expression for SOC-induced ISC rate contains a product of the FranckCondon weighted density of states, which depends on the $T_{1}-S_{0}$ energy separation and the shape of the $S_{0}$ potential energy surface, and the square of the matrix element of the SOC operator, $\left|\left\langle\mathrm{S}_{0}\left|\mathrm{H}_{\mathrm{so}}\right| \mathrm{T}_{1}\right\rangle\right|^{2}$. The same squared matrix element occurs in a formulation that explicitly considers molecular motion along a dynamic trajectory (ref. 7). Presently, we offer a qualitative non-mathematical formulation of the magnitude of $\left|\left\langle\mathrm{S}_{0}\left|\mathrm{H}_{\mathrm{so}}\right| \mathrm{T}_{1}\right\rangle\right|^{2}$ as a function of molecular structure, based (i) on a quantitative algebraic formulation of a simplified model (ref. 8), which is an update of the analysis provided by Salem and Rowland (ref. 9), and (ii) on results of $a b$ initio calculations (refs. 10,11) obtained with a version of GAMESS (ref. 12) that we modified using code from MELD (ref. 13), NBO (ref. 14), and SOCC (ref. 15).

\section{TRIPLET SUBLEVELS AND MOLECULAR MAGNETIC AXES}

In fact, there is not one value of $\left|\left\langle\mathrm{S}_{0}\left|\mathrm{H}_{\text {so }}\right| \mathrm{T}_{1}\right\rangle\right|^{2}$ but three, since the "triplet state" is a collection of three states very close in energy, and the reacting molecule can be in any one of them (ref. 16). The initial population of the sublevels may be uneven if they are reached by selective ISC from an excited singlet state. Even if the population is uniform initially, differential rates of ISC to $S_{0}$ may cause it to become nonuniform. Spontaneous equilibration of sublevel populations may take seconds at very low temperatures, but at room temperature, it occurs on a scale of ns or tens of ns. Indeed, a single exponential lifetime is often observed for the triplet, suggesting that the three sublevels are in rapid equilibrium. Then, individual values of the three squared matrix elements are not important and the square root of the sum of the three squares alone determines the rate of ISC to $S_{0}$. If the lifetime of the reacting triplet is too short, complete equilibration of sublevel populations may not be achieved even at room temperature, and individual values of the three squared matrix elements determine three independent ISC rate constants. 
In strong magnetic fields, the wave functions of the three spin sublevels of $T_{1}$ differ in the magnitude of the projection of their total electronic spin angular momentum into the direction of the field, and are labelled $T_{1,+1}, T_{1,0}$, and $T_{1,-1}$. However, in the absence of magnetic field, the wave functions differ in the projection of their total electronic spin into three mutually orthogonal magnetic axes $x, y, z$ in the molecular frame, and are labelled $\mathrm{T}_{1 x}, \mathrm{~T}_{1 y}$, and $\mathrm{T}_{1 z}$. We think of the three matrix elements, $\left\langle\mathrm{S}_{0}\left|\mathrm{H}_{\mathrm{so}}\right| \mathrm{T}_{1 x}\right\rangle,\left\langle\mathrm{S}_{0}\left|\mathrm{H}_{\mathrm{so}}\right| \mathrm{T}_{1 y}\right\rangle$, and $\left\langle\mathrm{S}_{0}\left|\mathrm{H}_{\mathrm{SO}}\right| \mathrm{T}_{\mathrm{iz}}\right\rangle$, as the components of a vector (the $\mathrm{S}_{0}-\mathrm{T}_{1}$ spin-orbit coupling vector). In the general ISC case, we need the length and the direction of this vector. For equilibrated sublevels, usually tacitly assumed, one only needs the length, $\left[\left.\left|<\mathrm{S}_{0}\right| \mathrm{H}_{\mathrm{so}}\left|\mathrm{T}_{\mathrm{lx}}\right\rangle\right|^{2}+\left|\left\langle\mathrm{S}_{0}\left|\mathrm{H}_{\mathrm{so}}\right| \mathrm{T}_{1 y}\right\rangle\right|^{2}+\left|\left\langle\mathrm{S}_{0}\left|\mathrm{H}_{\text {so }}\right| \mathrm{T}_{1 z}\right\rangle\right|^{2}\right]^{1 / 2}$.

The directions of the magnetic axes $x, y, z$ are dictated by the anisotropy of the distribution of the two unpaired spins in the molecule and by their spin magnetic dipole - magnetic dipole interaction, usually responsible for most of the energy differences between the three levels in the absence of magnetic field. These differences are known as the zero-field splitting (zfs), and are described by the EPR parameters $D$ and $E$. Unless the molecular symmetry is high, in which case the magnetic axes coincide with the symmetry axes, or unless only the length of the SOC vector is desired, a complete calculation of SOC needs to include a calculation of the spin dipole-dipole zfs tensor. If only the length of the SOC vector is desired, as was the case in most prior computations, any arbitrary choice of three directions $x, y, z$ will give the same result.

Before comparison with observed values, the zfs constants $D$ and $E$ obtained from the dipole-dipole interaction, and in the general case, also the direction of the magnetic axes, should be corrected for the effects of SOC, which mixes the three $T_{1}$ sublevels and nearby singlets and thus affects the relative energies of the triplet sublevels. The correction is normally negligible in organic biradicals, even if $S_{0}$ and $T_{1}$ are nearly degenerate, but in a molecule such as $\mathrm{SiH}_{2}$, with a heavier atom and a range of valence angles at which $\mathrm{T}_{1}$ and $\mathrm{S}_{0}$ cross, the calculated $D$ value is dominated by the SOC contribution (ref. 11).

\section{THE SPIN-ORBIT COUPLING (SOC) OPERATOR}

The SOC operator is a sum of a one-electron and a two-electron part. The one-electron part represents the energy of the interaction of the spin magnetic dipole moment of an electron with the magnetic dipole moment due to its orbital motion. The orbital moment is due to the acceleration imposed on the electron by nuclear attraction, and the operator is a sum of contributions from all nuclei. Those provided by nuclei with high charges $Z$, and therefore, high electric fields, are weighted disproportionately. For an electron contained in a singly occupied molecular orbital (MO) described as a linear combination of atomic orbitals (AOs), large contributions from a nucleus originate primarily from sojourn of the electron in AOs that are located at that nucleus and have a large angular asymmetry ( $p$, but not $s$ ). For protons $Z=1$, and nearby electrons sojourn primarily in $s$ orbitals, so $\mathrm{H}$ atoms contribute very little. Electrons in doubly occupied orbitals, such as inner shells, do not contribute at all since the contributions of the two opposed electron magnetic moments cancel. In a biradical well described as having two singly occupied MOs, SOC is due to electrons in these two orbitals.

The two-electron part corrects the one-electron part for the effect of nuclear shielding by electrons, particularly those in inner shells, and for the less important interaction of the spin magnetic moment of one electron with the orbital magnetic moment of another. E.g., it reduces the contributions of $\mathrm{C}$ atoms to about one half. The final result can be approximated by computing only the one-electron part and introducing effective atomic SOC constants $\zeta_{x}$, which correct for the effect of the two-electron part. They increase roughly as $Z_{k}{ }^{4}$. Our $a b$ initio computations use the full expression.

\section{THE SOC MATRIX ELEMENT}

The SOC operator couples triplet and singlet states, since it contains the product of a term that acts on the spin part of a wave function, converting a triplet function to a singlet function, and a term that acts on the space part of the wave function, changing one configuration to another. As noted above, the expression 
for a component of the SOC vector, $\left\langle\mathrm{S}_{0}\left|\mathrm{H}_{\mathrm{so}}\right| \mathrm{T}_{1 u}\right\rangle, u=x, y, z$, is a sum over all atoms. Each atom contributes a sum over pairs of all basis set orbitals in the molecule. Each term in the sum is multiplied by numerical coefficients obtained from the $\mathrm{CI}$ expansion and from the coefficients of valence (primarily p) orbitals in the two singly occupied MOs of $\mathrm{T}_{1}$, and reflects the degree to which a $90^{\circ}$ rotation around the axis $u$ through the atom converts one member of the orbital pair into the other. It is small unless both basis set orbitals are located on the atom in question ("one-center terms").

It is possible to use AOs for the definition of the basis set. In organic molecules, the three valence $p$ orbitals on each non-hydrogen atom will then play a critical role in understanding the origin of its contribution to the SOC vector. Since the two orbitals of each contributing pair need to be different, there are three distinct pairs on each atom $\left(p_{x}-p_{y}, p_{x}-p_{z}, p_{y}-p_{z}\right)$, and thus up to three important contributions. Since the choice of the molecular axes is unrelated to the local bonding situation, these $p$ orbitals will not necessarily have any simple interpretation. Also, reasonably large basis sets need to be used, and these will contain two or more sets of $p$ orbitals on each non-hydrogen atom, so the number of pairs to be considered will be greatly increased, and simple interpretation will be even more difficult. We avoid both problems by using a basis set of Weinhold's preorthogonalized natural hybrid orbitals (NHOs, ref. 14), which are directed along bonds and correspond to the only four valence hybrid orbitals drawn in simple pictures of bonding. They are rigorously defined even for large basis sets. We then need to consider six orbital pairs on each non-hydrogen atom. We had prior encouraging experience with NHOs in the interpretation of hyperfine coupling constants (ref. 17), which also depend on the delocalization of a singly occupied MO in a saturated skeleton. Another program for NHO analysis of SOC was developed independently by Zimmerman and Kutateladze (ref. 18).

\section{SOC IN BITOPIC BIRADICALS}

In bitopic biradicals the $S_{0}$ and $T_{1}$ states can be described by configurations with only two partially occupied MOs $A$ and $B$ and all other orbitals occupied either doubly or not at all. Orbitals $A$ and $B$ are chosen in their most localized form. E.g., in fully twisted ethylene, $A$ is located mostly on the free-radical $2 p$ orbital on one of the carbons, and is delocalized to a small extent onto the $\mathrm{CH}$ bonds of the other methylene, while $B$ is located mostly on the free-radical $2 p$ orbital of the other carbon and, to a small extent, on the $\mathrm{CH}$ bonds of the first methylene. $\mathrm{T}_{1}$ is approximated by a configuration with orbitals $A$ and $B$ singly occupied, and $\mathrm{S}_{0}$ by a linear combination of three: a dot-dot structure with $A$ and $B$ singly occupied by electrons of opposite spins $(A B)$, and the symmetrized and the antisymmetrized combinations of the two hole-pair structures with both electrons in the same orbital $A$ or $B\left(A^{2}+B^{2}\right.$ and $\left.A^{2}-B^{2}\right)$. Assuming that $A$ and $B$ are similar in the $S_{0}$ and $T_{1}$ states, the SOC vector can be approximated (ref. 8 ) by a particularly simple product of a scalar with a vector. The scalar is the coefficient of the symmetrized hole-pair structure $A^{2}+B^{2}$ in $\mathrm{S}_{0}$, and the vector is a sum of atomic vectors provided by non-hydrogen atoms.

\section{The Scalar Factor}

In a perfect biradical, whose two singly occupied most localized molecular orbitals $A$ and $B$ have equal energies and do not interact, the coefficient of the symmetrized hole-pair structure $A^{2}+B^{2}$ in the $\mathrm{S}_{0}$ state vanishes, and so does SOC (e.g., in orthogonally twisted ethylene). There are two independent ways to perturb a perfect biradical into a biradicaloid that can cause this coefficient to become non-zero, up to a limiting value of $1 / \sqrt{ } 2$. Either the energies of $A$ and $B$ become sufficiently different (e.g., in orthogonally twisted $\mathrm{H}_{2} \mathrm{BNH}_{2}$, a heterosymmetric biradicaloid), or $A$ and $B$ interact (have a non-zero resonance integral, e.g., in partially twisted ethylene, a homosymmetric biradicaloid). Both perturbations can act simultaneously (e.g., in partially twisted $\mathrm{H}_{2} \mathrm{BNH}_{2}$, a non-symmetric biradicaloid). Whether a difference in the energies of $A$ and $B$ is sufficient is determined by the nature of the initial perfect biradical. If it is of the axial type (ref. 19, e.g., carbene at a linear geometry), an arbitrarily small difference begins to make the coefficient of $A^{2}+B^{2}$ in the $\mathrm{S}_{0}$ state non-zero (e.g., bending of the carbene, which gives one of the orbitals some $s$ character). If it is of the pair biradical type (ref. 19, e.g., orthogonally twisted ethylene), the difference needs to be large (e.g., a protonated Schiff base), and a small difference does not help (e.g., orthogonally twisted propene). For additional detail, see ref. 8 . 


\section{Atomic Vectorial Contributions}

Each atomic vectorial contribution: (i) contains the factor $\zeta_{\mathrm{x}}$ (or its accurately computed analogue); (ii) is a sum of six principal terms, each due to a pair of distinct valence orbitals $\mu$ and $v$ on the atom (one-center contributions to SOC), and possibly some additional terms that originate from pairs of valence orbitals one of which is located on a neighbor atom (two-center contributions to SOC, usually much smaller), and (iii) has each of the six contributions multiplied by a factor $\left(c_{A \mu} c_{B v}-c_{A v} c_{B \nu}\right)$ containing the coefficients with which the orbitals of the $\mu v$ pair enter into $A\left(c_{A \mu} c_{A v}\right)$ and $B\left(c_{B v}, c_{B \nu}\right)$, and similarly for the usually small additional contributions that involve neighbor atoms (neglected in the algebraic model of ref. 8).

(i) The multiplicative entry of $\zeta_{\mathrm{x}}$ into the vectorial contribution from atom $\mathrm{k}$ causes the heavy atom effect. It is only important if factors (ii) and (iii) do not cause the atomic vectorial contribution to be small, and the location of the heavy atom in the molecule thus is of critical importance. If the vector provided by a heavy atom is not dominant and is directed against those provided by the other atoms, the introduction of the heavy atom may even reduce the total length of the resulting SOC vector (inverse heavy atom effect).

(ii) The vectorial contribution made by a valence NHO pair $\mu \nu$ is directed perpendicular to the plane defined by the axes of the hybrids $\mu$ and $\nu$. The sense of the vector is given by the direction of the left thumb when the curved fingers indicate the direction of rotation that converts $v$ into $\mu$. Its length is equal to the overlap of the $p$ part of the rotated NHO v with the NHO $\mu$. It is therefore proportional both to the percentage of $p$ character in the NHO $\mu$ and in the NHO v. It is unity for pure $p$ orbitals, and vanishes if one of the orbitals is of pure $s$ nature. In the usually small two-center contributions, with $\mu$ and $v$ located on different atoms, the overlap of the rotated NHO $v$ with the $\mathrm{NHO} \mu$ tends to be small, even if both are pure $p$ orbitals.

(iii) The multiplication of each of the hybrid orbital pair contributions by the factor $\left(c_{A \mu} c_{B v}-c_{A v} c_{B \mu}\right)$ guarantees that only those atoms that carry contributions from both singly occupied most localized orbitals $A$ and $B$ on two distinct hybrids can provide non-vanishing vectorial contributions: if a particular valence orbital pair $\mu \nu$ is to contribute, it is best for $\mu$ to appear in either $A$ or $B$, and $v$ in the other. This limits the number of contributing atoms and the number of contributing orbital pairs on any atom.

\section{Role of Symmetry}

Molecular symmetry may constrain the vectors provided by the various atoms and confine their sum to a single plane, or a single direction, or even cause it to vanish. This corresponds to situations in which one, two, or all three of the matrix elements $\left\langle\mathrm{S}_{0}\left|\mathrm{H}_{\text {so }}\right| \mathrm{T}_{1 u}\right\rangle, u=x, y, z$, vanish because of the symmetry properties of the wave functions of the $S_{0}$ and $T_{1 u}$ states. These follow easily from character tables once the symmetry properties of the singly occupied orbitals and thus those of the space part of the $S_{0}$ and $T_{1}$ wave functions are known, since the spin part of $T_{1 u}$ transforms like a rotation around $u$, while the spin part of $\mathrm{S}_{0}$ and the Hamiltonian $\mathrm{H}_{\text {so }}$ are totally symmetric (see ref. 6 for examples).

\section{GENERAL RULES FOR SOC IN BITOPIC BIRADICALS}

For large SOC between $S_{0}$ and $T_{1}$ of a bitopic biradical, the most localized orthogonal orbitals $A$ and $B$ singly occupied in $T_{1}$ should be as follows (ref. 8): (i) They either interact covalently through a nonzero resonance integral and/or are sufficiently different in energy for one of them to have electron occupancy near two in the $S_{0}$ state. (ii) The biradical contains one or more high- $Z$ atoms at which one $p$ orbital contributes strongly to $A$ and another to $B$. (iii) These $p$ orbitals enter into $A$ and $B$ in such a manner that the contributions on all such atoms add rather than cancel.

\section{ILLUSTRATIVE EXAMPLES}

We have chosen two heterosymmetric biradicaloids for illustration (Fig. 1): chlorocarbene, $\mathrm{HCCl}(1)$, and cis-chloromethylcarbene, $\mathrm{HCCH}_{2} \mathrm{Cl}$ (2), and computed the $\mathrm{SOC}$ vectors using $(2,2) \mathrm{MCSCF} / \mathrm{cc}-\mathrm{pVDZ}$. This level of calculation is adequate for the present purpose, but for quantitative work we normally use a larger 
active space. Geometrical variables were optimized for $T_{1}$, except for the valence angle $\phi$ at the divalent carbon, which was varied. The results shown here were obtained with $\phi=120^{\circ}$ for 1 (the optimized value is $124.5^{\circ}$ ), and with the optimized value of $130^{\circ}$ for 2 . Both molecules are planar, forcing one of the magnetic axes $(z)$ to lie perpendicular to the plane. A calculation of the spin-spin dipolar interaction reveals that the magnetic axis $x$ makes a $68^{\circ}$ angle with the $\mathrm{CCl}$ bond in 1 and a $67^{\circ}$ angle in 2 (Fig. 1 ; in a symmetrically substituted carbene this direction would bisect $\phi$ ). The $y$ axis is perpendicular to $x$ and $z$.

In 1 , the length of the SOC vector is $32.9 \mathrm{~cm}^{-1}$. The one-electron contribution is 49.7 and the two-electron contribution is $-16.8 \mathrm{~cm}^{-1}$. In 2 , the SOC vector length is $9.9 \mathrm{~cm}^{-1}$, with $21.4 \mathrm{~cm}^{-1}$ from the one-electron and $-11.5 \mathrm{~cm}^{-1}$ from the two-electron contribution (note that the total result is roughly equal to half the oneelectron result). In both species, the bitopic approximation is valid, and the results can be analyzed using the simple model. The $S_{0}$ wave function has almost no electron occupancy in the $\pi$-symmetry non-bonding atomic orbital on the divalent carbon, $A$, and almost full double occupancy in the o-symmetry non-bonding hybrid, $B$. The scalar factor is thus very favorable, $\sim 0.5$, close to its maximum possible value of $1 / \sqrt{ } 2$.

In 1 and 2 , the SOC vector is constrained to the symmetry plane, i.e., $\left\langle S_{0}\left|H_{S O}\right| T_{1 z}\right\rangle$ vanishes, and $T_{1 z}$ is not coupled to $S_{0}$ : the spin and space parts of the $S_{0}$ wave function are totally symmetric $\left(A^{\prime}\right)$, as is $H_{s o}$, while the space part of $T_{1}$ transforms like $A^{\prime \prime}$ (one $\sigma$ and one $\pi$ MO are singly occupied). Therefore, the spin part must also transform like $A^{\prime \prime}$ for the matrix element not to vanish. The spin function in $T_{1 u}$ transforms like a rotation around $u$. Rotation around $z$ is totally symmetric (A'), so the $z$ component of the SOC vector vanishes. Rotation around $x$ or $y$ transforms like $A^{\prime \prime}$, so the $x$ and $y$ components do not vanish.

In $\mathrm{CH}_{2}$ itself, symmetry is higher and the $\mathrm{SOC}$ vector $\left(13.1 \mathrm{~cm}^{-1}\right)$ is constrained to lie parallel to the $\mathrm{H}-\mathrm{H}$ line $(y$ axis). Only one of the six possible NHO pairs on carbon contributes significantly to $A, B$, and SOC.

In chlorocarbene 1, non-vanishing in-plane atomic vectorial contributions will be provided both by $\mathrm{C}$ and by $\mathrm{Cl}$, since both carry $A$ as well as $B$ in two different p orbitals: the $\pi$-symmetry non-bonding orbital $A$ is partially delocalized onto the $\mathrm{Cl} \pi$ lone-pair orbital by classical $\pi$ conjugation, and the $\sigma$-symmetry nonbonding orbital $B$ is partially delocalized onto the $\mathrm{Cl} \sigma$ lone-pair orbital, and also onto the hybrid used to make the $\mathrm{C}-\mathrm{Cl} \mathrm{o}$ bond, by o conjugation (Fig. 1). The degree of delocalization is small, and the orbital coefficients much larger on $\mathrm{C}(0.97,0.92)$ than on $\mathrm{Cl}(-0.23,-0.21,-0.16)$. The atomic vectorial contribution on $C\left(8.8 \mathrm{~cm}^{-1}\right)$ is somewhat reduced relative to carbene, and lies in nearly the same direction. The coefficients of $A$ and $B$ on $\mathrm{Cl}$ are small enough that its contribution would be negligible if it were not for its large $Z$. Only two of the six contributions are significant, and the atomic vector is perpendicular to the axes of the $\mathrm{Cl}$ atomic hybrids that enter $A$ and $B$ (its length is $25.5 \mathrm{~cm}^{-1}$ ). The relative phases on these hybrids in these two MOs are the same on $\mathrm{C}$ and on $\mathrm{Cl}$, so the sense of the two atomic vectors is the same. The vectors are not quite parallel, and the length of the sum is a little less than the sum of their lengths.
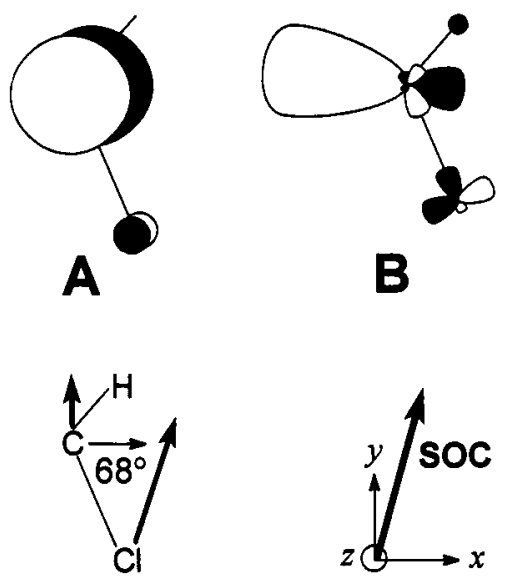
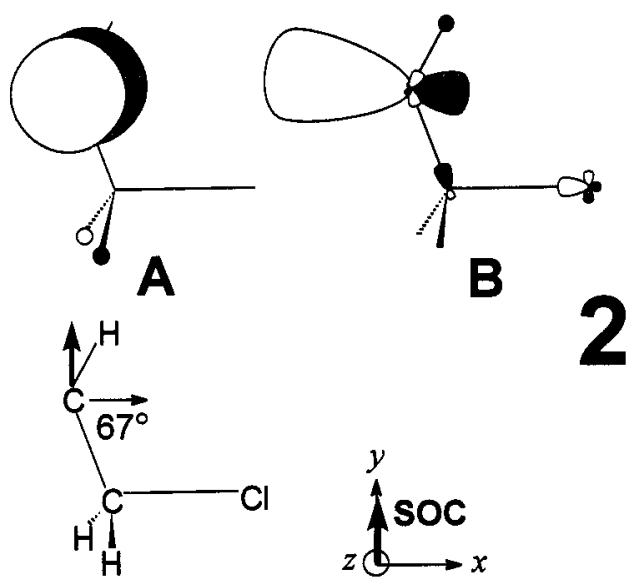

Figure 1. Open-shell orbitals $A$ and $B$ (schematic) and atomic vectorial contributions to SOC in $\mathbf{1}$ and 2. 
This example illustrates the origin of the heavy atom effect as due to minor delocalization of the open-shell orbitals $A$ and $B$ onto a high- $Z$ atom, provided that they delocalize onto different $p$ orbitals. It also illustrates the vectorial nature of the addition of atomic contributions. Our second example, chloromethylcarbene (2), demonstrates a situation in which the introduction of a $\mathrm{Cl}$ atom has virtually no effect on the SOC vector, since only one of the open-shell orbitals delocalizes onto it. In 2 , the $\pi$-symmetry non-bonding orbital $A$ is weakly delocalized onto the $\mathrm{CH}_{2}$ group by $\pi$ hyperconjugation, but the delocalization does not reach as far as the $\mathrm{Cl} \pi$ lone-pair orbital. The $\sigma$-symmetry non-bonding orbital $B$ is quite significantly delocalized onto the $\mathrm{Cl}$ hybrid used to make the $\mathrm{C}-\mathrm{Cl} \sigma$ bond (coefficient 0.17 ), by antiperiplanar o conjugation (Fig. 1), but in itself, this is not sufficient. Not surprisingly, the atomic contribution to the SOC vector provided by the $C$ atom, $9.9 \mathrm{~cm}^{-1}$, is still close to what it was in carbene itself. However, in spite of its high $Z$, the $\mathrm{Cl}$ atom contributes only $0.5 \mathrm{~cm}^{-1}$ to the SOC vector, because only one of the open-shell orbitals reaches this atom to a significant extent.

\section{CONCLUSION}

The effects of biradical structure on SOC can be computed and understood in terms of a set of simple rules, providing a predictive tool for triplet photochemistry, and a means for analyzing orbital delocalization in both saturated and unsaturated systems. The presently illustrated dominance of through-bond coupling over through-space coupling mediated by two-center terms seems to be general (refs. 2,8,18,20,21), and permits further insight in terms of standard resonance structures (ref. 8).

Acknowledgement. This work was supported by the US NSF (CHE-9318469 and CHE-9412767).

\section{REFERENCES}

1. N. J. Turro, Modern Molecular Photochemistry. University Science Books, Mill Valley, CA (1991); A. Gilbert and J. Baggott, Essentials of Molecular Photochemistry. CRC Press, Boca Raton, FL (1991).

2. J. Michl and V. Bonaxic-Koutecký, Electronic Aspects of Organic Photochemistry. Wiley, New York, NY (1990); M. Klessinger and J. Michl, Excited States and Photochemistry of Organic Molecules. VCH, New York, NY (1995).

3. R. A. Caldwell, L. D. Jacobs, T. R. Furlani, E. A. Nalley, and J. Laboy, J. Am. Chem. Soc. 114, 1623 (1992).

4. N. J. Turro, G. Kavarnos, V. Fung, A. L. Lyons, Jr. and T. Cole, Jr., J. Am. Chem. Soc. 94, 1392 (1972).

5. G. L. Closs, M. D. E. Forbes, and P. Piotrowiak, J. Am. Chem. Soc. 114, 3285 (1992).

6. W. G. Richards, H. P. Trivedi, and D. L. Cooper, Spin-Orbit Coupling in Molecules. Clarendon Press, Oxford (1981); S. P. McGlynn, T. Azumi, and M. Kinoshita, Molecular Spectroscopy of the Triplet State. Prentice-Hall, Englewood Cliffs, NJ (1969); R. L. Ellis and H. H. Jaffe. In Modern Theoretical Chemistry (G. Segal, ed.). Plenum Press, New York, NY, Vol. 8, p. 74.

7. A. Morita and S. Kato, J. Phys. Chem. 97, 3298 (1993).

8. J. Michl, J. Am. Chem. Soc. 118, 3568 (1996).

9. L. Salem and C. Rowland, Angew. Chem., Int. Ed. Engl. 11, 92 (1972).

10. Z. Havlas and J. Michl, submitted for publication.

11. Z. Havlas, J. W. Downing, and J. Michl, unpublished results.

12. M. Dupuis, D. Spangler, and J. J. Wendoloski, Program QG01, National Resource for Computations in Chemistry, Software Catalog, University of California, Berkeley, CA (1980); M. W. Schmidt, K. K. Baldridge, J. A. Boatz, S. T. Elbert, M. S. Gordon, J. H. Jensen, S. Koseki, N. Matsunaga K. A. Nguyen, S. J. Su, T. L. Windus, M. Dupuis, and J. A. Montgomery, J. Comput. Chem. 14, 1347 (1993).

13. E. R. Davidson and D. Feller, MELD, Indiana Universtity, Bloomington, IN, USA (version distributed in METECC 94).

14. E. D. Glendening, J. K. Badenhoop, A. E. Reed, J. E. Carpenter, and F. Weinhold, NBO 4.0. Theor. Chem. Inst., University of Wisconsin, Madison, WI (1994); A. E. Reed, L. A. Curtis, and F. Weinhold, Chem. Rev. 88, 899 (1988).

15. T. R. Furlani, Ph.D. Dissertation, State University of New York at Buffalo, 1985; T. R. Furlani and H. F. King, J. Chem. Phys. 82, 5577 (1985).

16. A. Carrington and A. D. McLachlan, Introduction to Magnetic Resonance. Harper and Row, New York (1967); W. Weltner, Jr, Magnetic Atoms and Molecules. Van Nostrand Reinhold, New York (1983).

17. A. J. McKinley, P. N. Ibrahim, V. Balaji, and J. Michl, J. Am. Chem. Soc., 114, 10631 (1992).

18. H. E. Zimmerman, A. G. Kutateladze, Y. Maekawa, and J. E. Mangette, J. Am. Chem. Soc. 116, 9795 (1994); H. E. Zimmerman and A. G. Kutateladze, J. Org. Chem. 60, 6008 (1995); J. Am. Chem. Soc. 118, 249 (1996).

19. V. Bonačić-Koutecký, J. Koutecký, and J. Michl, Angew. Chem. Internat. Ed. Engl. 26, 170 (1987); J. Michl and V. Bonaxić-Koutecký, Tetrahedron 44, 7559 (1988); J. Michl, J. Mol. Struct. (Theochem.) 260, 299 (1992).

20. L. Carlacci, C. Doubleday, Jr., T. R. Furlani, H. F. King, and J. W. Mclver, Jr., J. Am. Chem. Soc.109, 5323 (1987).

21. Michl, J.; Downing, J. W., 5th IAPS Winter Conference, Clearwater Beach, FL, 2-6 Jan. 1993, Abstract C3. 